\title{
Male gender and duration of anti-tuberculosis treatment are associated with hypocholesterolemia in adult pulmonary tuberculosis patients in Kampala, Uganda
}

\author{
John Mukisa ${ }^{1,2}$, Ismael Kawooya ${ }^{3}$, Joan Nangendo ${ }^{1}$, Annet Nalutaaya ${ }^{3}$, Jean Nyamwiza ${ }^{1}$, \\ Ali Sam ${ }^{1}$, Ronald Ssenyonga ${ }^{1}$, William Worodria ${ }^{4}$, Ezekiel Mupere ${ }^{5}$
}

1. Makerere University College of Health Sciences, clinical Epidemiology Unit.

2. Uganda CWRU Research Collaboration, clinical care.

3. Makerere University College of health sciences, medicine.

4. Mulago Hospital, Medicine.

5. College of Health Sciences Makerere University, Paediatrics \& Child Health; Clinical Epidemiology Unit.

\begin{abstract}
Background: Patients with Pulmonary tuberculosis (PTB) and hypocholesterolemia have an altered immune function, delayed sputum conversion at two months and increased mortality. However, the assessment for dyslipidemias is not often done in our setting.

Methods: A cross-sectional study was conducted among adults at an urban TB clinic in Kampala, Uganda. We included different participants at diagnosis (0), 2, 5, 6 and 8 months of anti-TB treatment. Data was collected from a complete physical examination, a pre-tested structured questionnaire, six-hour fasting lipid profiles and random blood glucose levels.

Results: Of the 323 included participants, 63.5\% (205/323) were males and the median age was 30 years, IQR (23-39). The prevalence of hypocholesterolemia was $43.65 \%$ (95\% CI 38.3-49.2). The participants at diagnosis had the highest hypocholesterolemia prevalence, $57.3 \%, 95 \%$ CI (46.7-67.2); and lowest amongst those completing treatment at 6/8 months, 32.2\%, 95\% CI (21.6-45.2). Significant factors associated with hypocholesterolemia were: male gender (PR 1.52, 95\% CI: 1.13-2.03), and duration of anti-TB treatment (0.88, 95\% CI: 0.80-0.98).

Conclusion: Hypocholesterolemia is common among patients with PTB. The risk of hypocholesterolemia increases with being male and reduces with increased duration of treatment. There is a need for further research in lipid abnormalities in TB patients. Keywords: Hypocholesterolemia, pulmonary tuberculosis, duration of anti-tuberculosis treatment, gender.

DOI: https://dx.doi.org/10.4314/ahs.v18i3.3

Cite as: Mukisa J, Kawooya I, Nangendo J, Nalutaaya A, Nyamwiza J, Sam A, Ssenyonga R, Worodria W, Mupere E. Male gender and duration of anti-tuberculosis treatment are associated with hypocholesterolemia in adultpulmonary tuberculosis patients in Kampala, Uganda. Afri Health Sci. 2018;18(3): 479-487. https://dx.doi.org/10.4314/abs.v18i3.3
\end{abstract}

\section{Introduction}

Pulmonary Tuberculosis (PTB) is still a major global health problem estimated to cause 10.4 million new cases

\section{Corresponding author: \\ John Mukisa, \\ Makerere University College of Health Sciences, clinical Epidemiology Unit; \\ Uganda CWRU Research Collaboration, clinical care. Email: jmukisa90@gmail.com, mukisajohn90@gmail.com}

and 1.8 million related deaths annually ${ }^{1,2}$. Africa bears about $25 \%$ of the world's PTB cases and yet it has the lowest number of health professionals, with the majority of sub-Saharan Africa countries like Uganda having less than 5 physicians per 1000 population ${ }^{3}$. The incidence of PTB in Africa is estimated at 280 per 100,000 compared to the global incidence of 126 per 100000 . In comparison, Uganda has an estimated incidence of TB at 202/100000 population ${ }^{2}$.

PTB patients often have deranged serum lipid levels, especially low cholesterol levels (hypocholesterolemia) which are an important cause of morbidity ${ }^{4}$. The hypo- 
cholesterolemia in PTB patients has been shown to alter the body immunity, and could possibly be associated with delayed sputum conversion and increased mortality especially in circumstances of drug resistance ${ }^{5}$.

Lipid abnormalities in PTB patients are associated with Anti-retroviral therapy (ART) especially Non-Nucleoside Reverse Transcriptase Inhibitors (NNRTIs) and Protease Inhibitors (PIs), HIV, malnutrition, cavitary disease on the $\mathrm{X}$-ray and older age ${ }^{6-9}$. However, the characterization of lipid abnormalities in sub-Saharan Africa which has a high prevalence of HIV and malnutrition is not well documented $^{10,11}$. In Uganda, the WHO and National TB and Leprosy Programme (NTLP) recommend clinical, bacteriological, nutritional assessment and monitoring of $\mathrm{TB}$ patients at diagnosis and throughout the course of treatment but this is rarely done and if performed only stops at anthropometric measurements ${ }^{12,13}$. This paper, therefore, presents the prevalence and factors associated with hypocholesterolemia among adult PTB patients either at diagnosis, 2, 5 and $6 / 8$ months of anti-TB treatment at an urban out-patient clinic located in Kampala, Uganda.

\section{Methods \\ Design and setting}

This was a cross-sectional study conducted at the Uganda National TB and Leprosy Programme (NTLP) Clinic, Mulago National Referral and Teaching Hospital Complex between February and April 2016.

Mulago hospital serves patients from all over Uganda and residents of the surrounding areas in Kampala district. The NTLP clinic serves about 60-80 patients per month of whom a quarter are new patients. The number of patients bacteriologically confirmed each month varies depending on the test used, with about 60-65, 20-28 and 6-12 patients confirmed using Gene Xpert,sputum smear, and solid culture respectively. The clinic provides out-patient and in-patient care to PTB patients of all categories including new, relapse, and continuation phase. Patients are followed up regularly as per national treatment guidelines that is to say at diagnosis (0), 2, 5, 6, 8 months after starting treatment and wherever it is necessary. Patient follow-up clinic visits entail drug refills, consultation, and management of any arising complications including adverse effects according to the national TB program guidelines ${ }^{13}$.

\section{Eligibility criteria}

We included all adults ( $\geq 18$ years) with PTB at either: diagnosis (0) or at 2, 5, 6 /8 months of treatment. Mycobacterium tuberculosis was bacteriologically confirmed either with Gene Xpert, Sputum smear, or culture. For participants at diagnosis, their current diagnostic result was considered. Among those at 2, 5, 6/ 8 months of treatment, their patient charts were retrieved and initial diagnostic result before starting treatment was considered before enrollment into the study. All participants provided signed informed consent before enrolment into the study. We excluded participants taking any lipid-lowering drugs for example statins and fibrates, and those who were very ill with the kanorffsky score less than $20 \%$ at the time of enrollment.

\section{Sample size calculation}

We estimated a sample size of 427 adults using the Kish Leslie formula ${ }^{14}$, assuming a $50 \%$ prevalence rate, 0.05 precision level, and factoring for $10 \%$ non-response.

\section{Sampling procedure}

The participants were consecutively enrolled with no stratification according to the duration of treatment.

\section{Data collection}

Study procedures included a complete physical examination, blood draws for laboratory tests (lipid profile, random blood sugar) and administration of a pre-tested structured questionnaire. Information collected on the questionnaire included socio-demographic characteristics, nutritional status, co-morbid illnesses, socio-economic characteristics, and lifestyle and clinical factors.

We drew $4 \mathrm{mls}$ of venous blood samples from the ante-cubital fossa for each participant, 6 hours after the last meal to assess the lipid profile and fasting blood sugar. The samples for lipid profiles were analyzed using COBAS 6000 (Roche Diagnostics Ltd) at Mulago Hospital clinical chemistry laboratory. A Fasting blood sugar was

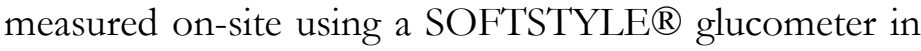
accordance with standard operating procedures. All participants included in the study had undergone a test for HIV infection following the national HIV serial testing algorithm ${ }^{15}$.

We also took anthropometric measurements such as height and weight. Weight was taken using an adult SECA 
digital electronic scales to the nearest $100 \mathrm{~g}$. Height was measured to the nearest $\mathrm{cm}$ of standing height using a stadiometer. All anthropometric measurement values were means of duplicates. Body Mass Index (BMI) was calculated as weight divided by height in meters squared. The participants recruited at diagnosis had a posteroanterior chest X-ray taken as per national guidelines. For patients enrolled at 2, 5, 6 or 8 months of treatment, their initial chest $\mathrm{X}$-rays at diagnosis were retrieved from the repository and read.

\section{Data management and analysis plan}

Data were double entered into Epidata version 3.1 and analyzed using STATA version 12.0 (Stata Corp College Station TX, USA). Categorical variables were summarized using proportions while means, median and inter-quartile range were used to summarize continuous variables. The prevalence of hypocholesterolemia was defined as the proportion of individuals with total serum cholesterol levels less than $3.7 \mathrm{mmol} / \mathrm{l}$ amongst the total number of participants included in the study. A Diagnosis of diabetes mellitus was made if a participant had a six-hour fasting blood sugar $\geq 126 \mathrm{mg} / \mathrm{dl}^{16}$.

We estimated prevalence ratios in bivariate and multivariate analyses using a generalized linear model with a $\log$ identity and binomial link. Participants with hyper- cholesterolemia (total serum cholesterol levels more than $5.7 \mathrm{mmol} / \mathrm{l}$ ), were excluded from further analysis in assessing for factors associated. Clinical significance and a cut-off $P$ value of $\leq 0.25$ during bivariate analyses were used to select variables for multivariate analysis ${ }^{9,17,18}$. Twoway product terms were formed among the variables in the model to assess for interaction using the chunk test. We also assessed for confounding by checking for a 10\% change in the effect measure.

\section{Ethical consideration}

Ethical approval was obtained from the Makerere University School of Medicine Research and Ethics Committee (\#REC REF 2016-017). All patients provided written informed consent. All study data were kept under lock and key to maintain privacy and confidentiality. We also referred all participants with hypocholesterolemia to clinicians for nutritional counseling.

\section{Results}

\section{Description of the study population}

Of the 371 participants screened, 323 were included in the study (Figure 1). Most of the participants were male $(63.2 \%)$, and Catholics (35.9\%). The median age of the participants was 30 years, Inter Quartile Range (23-39), (Table 1).

Figure 1: The profile of TB patients enrolled in the study at Mulago Hospital TB ward, February-April 2016

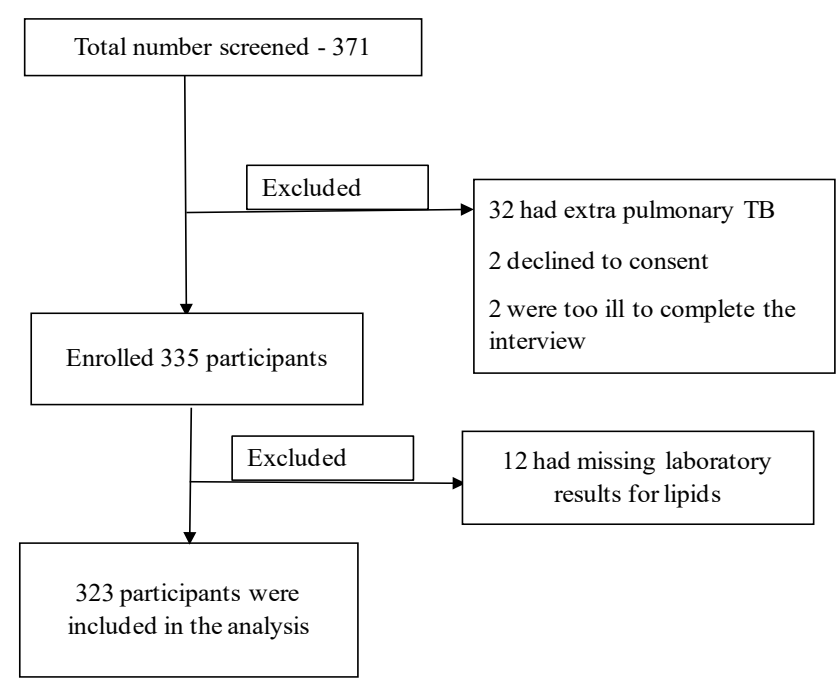


Table 1. Socio-demographic characteristics of the study participants with PTB at Mulago Hospital, Kampala February -April 2016 (N=323)

\begin{tabular}{lcc}
\hline Characteristic & Number & Percentage (\%) \\
\hline Gender & 205 & \\
Male & 118 & 63.5 \\
Female & & 36.5 \\
& & \\
Religion & 93 & 28.8 \\
Protestant & 115 & 35.6 \\
Catholic & 66 & 20.4 \\
Moslem & 40 & 12.4 \\
Pentecostal & 7 & 2.2 \\
Seventh Day Adventist & 2 & 0.6 \\
Others* & & \\
& 144 & 44.6 \\
Distance from clinic & 179 & 55.4 \\
< 5km & & \\
>=5 km & 117 & 36.2 \\
& 72 & 22.3 \\
Marital status & 134 & 41.5 \\
Married & & \\
/Divorced & & 65.0 \\
Single & 138 & 35.0 \\
Occupation & 52 & \\
Employed** & & \\
None & 113 & 16.1 \\
Level of education & & \\
None & 210 & \\
Primary & & \\
Secondary & & \\
Tertiary level & & \\
& & \\
\hline
\end{tabular}

*included those who had no religion, traditionalist

**includes self -employed, salaried employee and peasant farmers 
About $64.1 \%$ of participants had normal BMI (18.5- ties on chest X-ray (Table 2). About $95.2 \%$ of the HIV 24.5), $31.9 \%$ were HIV positive and $27.9 \%$ had cavi-

positive participants were currently taking anti-retroviral drugs.

Table 2. Clinical characteristics of the study participants with bacteriologically confirmed TB at diagnosis and during Anti TB treatment at Mulago Hospital Kampala, February April 2016. ( $\mathrm{N}=323)$

\begin{tabular}{|c|c|c|}
\hline Characteristic & Frequency & Percentage \\
\hline \multicolumn{3}{|l|}{ Duration of Anti-TB treatment } \\
\hline Diagnosis & 89 & 27.6 \\
\hline 2 months & 93 & 28.8 \\
\hline 5 months & 82 & 25.4 \\
\hline $6 / 8$ months & 59 & 18.2 \\
\hline \multicolumn{3}{|l|}{ HIV infection status } \\
\hline Positive & 103 & 31.9 \\
\hline Negative & 220 & 68.1 \\
\hline \multicolumn{3}{|l|}{ Confirmation of TB disease } \\
\hline \multicolumn{3}{|l|}{ Sputum Smear grade } \\
\hline AFB $1+$ & 58 & 17.9 \\
\hline AFB 2+ & 52 & 16.1 \\
\hline AFB $3+$ & 46 & 14.2 \\
\hline Gene Xpert & 157 & 48.7 \\
\hline Sputum culture* & 10 & 3.1 \\
\hline \multicolumn{3}{|l|}{ Body mass index(BMI) } \\
\hline$<18.5$ & 82 & 25.4 \\
\hline $18.5-24.5$ & 207 & 64.1 \\
\hline$>24.5$ & 34 & 10.5 \\
\hline \multicolumn{3}{|l|}{ Chest X-ray } \\
\hline Cavities & 89 & 27.9 \\
\hline No cavities $* *$ & 230 & 72.1 \\
\hline \multicolumn{3}{|l|}{ Diabetes Mellitus } \\
\hline No & 283 & 87.6 \\
\hline Yes & 40 & 12.38 \\
\hline \multicolumn{3}{|l|}{ ART use $(n=103)$} \\
\hline Currently on ARVs & 98 & 95.2 \\
\hline Not on ARVs & 5 & 4.8 \\
\hline \multicolumn{3}{|l|}{ Type of ARVs(n=98) } \\
\hline Protease Inhibitors & 3 & 3.0 \\
\hline NNRTIs & 95 & 97.0 \\
\hline \multicolumn{3}{|l|}{ Smoking status } \\
\hline ever smoked & 54 & 16.7 \\
\hline Never smoked & 269 & 83.3 \\
\hline \multicolumn{3}{|l|}{ Alcohol intake } \\
\hline Currently drink & 52 & 16.1 \\
\hline Do not drink & 271 & 83.9 \\
\hline \multicolumn{3}{|l|}{ Hypertension } \\
\hline No & 287 & 88.9 \\
\hline Yes & 36 & 11.1 \\
\hline
\end{tabular}


Prevalence of hypocholesterolemia and associated factors.

The prevalence of hypocholesterolemia among the study participants was $43.6 \%$ (95\% CI: 38.3-49.2). This was highest amongst those at diagnosis (57.3\%) and lowest $(32.2 \%)$ among those at $6 / 8$ months that is to say the end of treatment (Table 3).

\section{Table 3. Proportions of participants with cholesterol abnormalities at diagnosis and during anti- TB treatment among PTB adults attending Mulago Hospital, February-April, 2016}

\begin{tabular}{|c|c|c|c|c|c|}
\hline $\begin{array}{l}\text { Total serum } \\
\text { cholesterol }\end{array}$ & Diagnosis $(n=89)$ & $\begin{array}{l}2 \text { months } \\
(n=93)\end{array}$ & 5 months ( $n=82$ ) & $6 / 8$ months ( $n=59)$ & $\begin{array}{l}\text { Total } \\
(\mathrm{N}=323)\end{array}$ \\
\hline \multirow{2}{*}{$\begin{array}{l}\text { Low levels } \\
\text { ( }<3.7 \mathrm{mmol} / \mathrm{l}), \mathrm{n} \\
\text { (proportion,95 } \\
\% \mathrm{Cl} \text { ) }\end{array}$} & 51 & 40 & 31 & 19 & 141 \\
\hline & $(57.3,46.76-67.22)$ & $(43.0,33.28-53.32)$ & $(37.8,27.92-48.8)$ & $(32.2,21.46-45.23)$ & $(43.65,38.31-\quad 49.15)$ \\
\hline \multirow{2}{*}{$\begin{array}{l}\text { Normal (3.7- } \\
57 \mathrm{mmol} / 1), \mathrm{n} \\
\text { (proportion,95 } \\
\% \mathrm{Cl} \text { ) }\end{array}$} & 35 & 47 & 48 & 34 & 164 \\
\hline & $(39.33,29.67-49.89)$ & $(50.5,40.40-60.63)$ & $(58.5,47.53-68.75)$ & $(57.6,44.64-69.64$ & $(50.8,45.31-56.22)$ \\
\hline \multirow{2}{*}{$\begin{array}{l}\text { High }(>5.7 \\
\text { mmol/1),n } \\
\text { (proportion,95 } \\
\% \mathrm{Cl} \text { ) }\end{array}$} & 3 & 6 & 3 & 6 & 18 \\
\hline & $(3.3,1.07-10.03)$ & $(6.6,2.91-1.37)$ & $(3.7,1.17-10.85)$ & $(10.2,4.59-21.02)$ & $(5.6,5.53-8.69)$ \\
\hline
\end{tabular}

The Bivariate analysis showed that having diabetes mellitus was associated with a $44 \%$ increased risk of hypocholesterolemia (PR: 1.44, $95 \%$ CI $(1.09,1.90)$ compared to those without diabetes mellitus. Being greater than 30 years old increased the risk of having hypocholesterolemia by $9 \%$ (PR: $1.09,95 \%$ CI $(0.86,1.40)$ when compared to those less than 30 years. The participants who were HIV positive were 0.86 times less likely (PR: 0.86, $95 \%$ CI (0.65-1.13) to have hypocholesterolemia when compared to their HIV sero-negative counterparts (Table 4). HIV status, gender, diabetes, duration of anti-TB treatment and the presence of cavities were considered for a series of multivariate models based on previous studies, clinical significance and P-value less than 0.25. HIV status, diabetes, and presence of cavities were also considered for assessment of interaction and confounding based on biologic plausibility and previous studies ${ }^{7,8}$. None of these variables had any significant interaction terms nor meaningful confounding effects. The final model had two covariates (Table 4). Being male was associated with a $52 \%$ statistically significant increase in the prevalence of hypocholesterolemia compared to females $(\mathrm{PR}=1.52$ 95\% CI: (1.13-2.03), P=0.005). Participants who had had 5 months of treatment were $30 \%$ less likely to have hypocholesterolemia ( $\mathrm{PR}=0.71$ 95\% CI: (0.51-0.97), $\mathrm{P}=0.033)$ when compared to those who were at diagnosis (not yet started treatment). Similarly, participants who were at $6 / 8$ months of treatment were 35\% (PR 0.65, 95\% CI: (0.440.97), $\mathrm{P}=0.033$ ) less likely to have hypocholesterolemia when compared to those who were at diagnosis. 
Table 4. Regression analysis of factors associated with hypocholesterolemia among adults with PTB at Mulago Hospital, February -April 2016. ( $\mathbf{N = 3 0 5 )}$

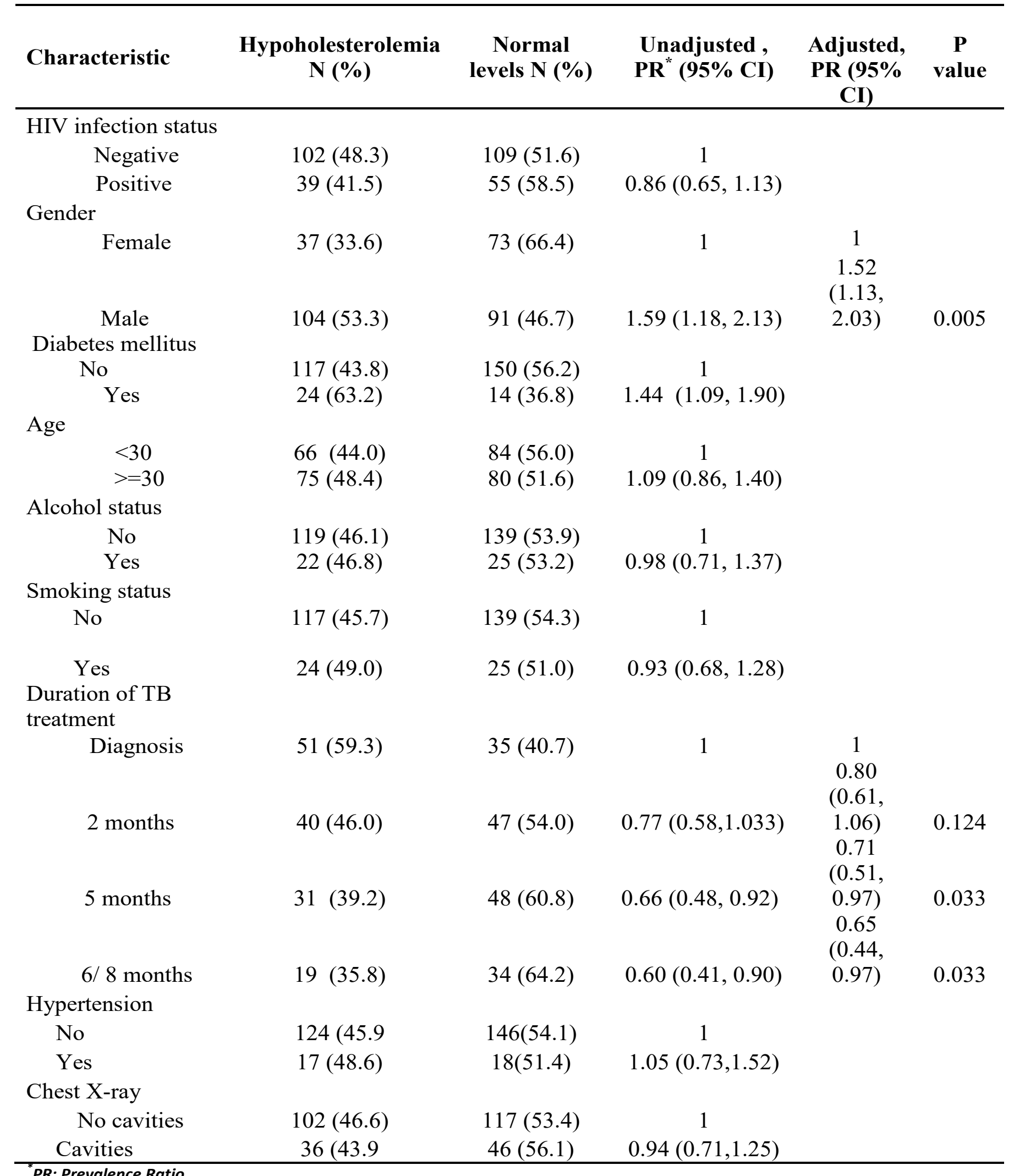




\section{Discussion}

Nearly half of the participants in the study population had hypocholesterolemia. This is among the first studies to document the prevalence of hypocholesterolemia in TB patients in Uganda.

We found a high prevalence of hypocholesterolemia among the study participants with the highest proportion being detected among those at diagnosis and lowest among those who had received 6/8 months of treatment. The high proportions of hypocholesterolemia at diagnosis may be due to inflammation caused by PTB which may worsen as the duration of symptoms and severity of the disease increases ${ }^{19,20}$. Also, at diagnosis, hypocholesterolemia proportions may be a consequence of the TB disease although it cannot be elucidated whether it is a factor contributing to the development of the active disease $^{4}$. In our study, participants who were at 5 months of anti-TB treatment had slightly higher proportions of hypocholesterolemia when compared to those who were at $6 / 8$ months of treatment $(37.8 \%$ versus 32.2 $\%$ respectively. This is contrary to the expected close proportions of hypocholesterolemia in the two groups. This may be due to the fact that those who were at 6/ 8 months of treatment received the anti-TB drugs for a longer duration and may have received different types of drug regimens. The lowered hypocholesterolemia proportions among participants at $6 / 8$ months (end of TB treatment) may possibly be explained by better response to treatment with improved appetite and increased nutrient intake especially of cholesterol-rich foods like eggs and fish.

The study results demonstrated that duration of anti TB treatment was associated with hypocholesterolemia. This may be due to the fact that the longer an individual is adherent to TB medications, the more their immunity is improved leading to reduced metabolic disturbances and improved appetite ${ }^{21}$. This improvement leads to nutritional recovery during the course of anti-TB treatment.

Males were $52 \%$ more likely to develop hypocholesterolemia as compared to females. This finding may possibly be attributed to the hormonal differences, reduced immunity and reduced nutrient intake especially fats which may occur differentially among men and women with
$\mathrm{PTB}^{21}$. The varying poor nutrient uptake in male PTB patients may probably be mediated by inflammatory markers released during TB disease (especially tumor necrosis factor-alpha) which interacts with human metabolic pathways that lead to anorexia ${ }^{22}$. Furthermore, males in our study presented with more severe disease (cavities on chest X-ray) possibly leading to a higher predisposition to hypocholesterolemia ${ }^{23}$.

The strength of this study hinges on the fact that we considered participants at different time points of treatment which gives a broad spectrum of dyslipidemias among TB patients although a cohort study with larger sample size and a homogenous group of study participants followed through time would be the ideal.

The interpretation of findings from this study should be made with caution as it had some limitations. First, we studied different sub-populations at varying time points of TB treatment. There could be underlying low levels of total serum cholesterol and thus the observed increase in mean total serum cholesterol concentrations as the duration of anti TB treatment may differ if the same individuals are followed up over time during care. Further misclassification bias was minimized by training of the research assistants, pre-testing the questionnaire before data collection, calibration of the weighing scale and stadiometer daily.

It is also important to note that this being a cross-sectional study, causality could not be assessed. However, we recruited participants that had taken treatment at varying time points to mimic a cohort study in temporality. Selection bias which may have arisen from referral bias since Mulago hospital is a tertiary referral center was minimized by recruiting participants from the catchment area of the population. The findings from this study are generalizable to adults with PTB in African settings seeking care at tertiary level health centers.

\section{Conclusion}

The overall prevalence of hypocholesterolemia among adult PTB patients presenting at Mulago NTLP clinic in Kampala was high. Being male and duration of anti-Tuberculosis treatment were significantly associated with hypocholesterolemia. The findings from our study may need further evaluation in longitudinal studies employing 
larger sample sizes to determine the effect of low total serum cholesterol levels on the parameters used to assess response to anti-TB treatment like sputum conversion, cure, and mortality.

\section{Conflict of interest}

All authors declare no conflict of interest.

\section{References}

1. Dye C, Scheele S, Dolin P, et al. Global burden of tuberculosis: estimated incidence, prevalence, and mortality by country. JAMA. 1999;282(7):677-686. PubMed.

2. WHO. Global Tuberculosis Report 2016.

3. WHO.Available:http:/ / www.who.int/gho/ health_workforce/physicians_density/en/.Accessed 25/09/2017, 2017.

4. Pérez-Guzmán C, Vargas MH. Hypocholesterolemia: A major risk factor for developing pulmonary tuberculosis? Medical hypotheses. 2006;66(6):1227-1230. PubMed.

5. Kozarevic D, McGee D, Vojvodic N, et al. Serum cholesterol and mortality: the Yugoslavia Cardiovascular Disease Study. Am J Epidemiol. 1981;114(1):21-28. PubMed.

6. Daniyam C, Iroezindu M. Lipid Profile of Anti-Retroviral Treatment-Naïve HIV-Infected Patients in Jos, Nigeria. Ann Med Health Sci Res. 2013;3(1):26-30.

7. Riddler SA, Smit E, Cole SR, et al. Impact of HIV infection and HAART on serum lipids in men. JAMA. 2003;289(22):2978-2982. PubMed.

8. Deniz O, Gumus S, Yaman H, et al. Serum total cholesterol, HDL-C and LDL-C concentrations significantly correlate with the radiological extent of disease and the degree of smear positivity in patients with pulmonary tuberculosis. Clinical Biochemistry. 2007;40:162-166.

9. Padmapriyadarsini C, Ramesh Kumar S, Terrin N, et al. Dyslipidemia among HIV-infected Patients with Tuberculosis Taking Once-daily Non-nucleoside Reverse-Transcriptase Inhibitor-Based Anti-retroviral Therapy in India. Clin Infect Dis. 2011;52(4):540-546.

10. Ni WQ, Liu XL, Zhuo ZP, et al. Serum lipids and associated factors of dyslipidemia in the adult population in Shenzhen. Lipids Health Dis. 2015;14.

11. Macallan DC. Malnutrition in Tuberculosis. Diagn Microbiol Infect Dis. 1999;34(2): 1153-1157.

12. WHO.Treatment of Tuberculosis guidelines World Health Organisation 2010.

13. MoH. Ministry of Health Manual of the National Tuberculosis and Leprosy Programme In Health Mo, (Ed) 2010.

14. Kish L. Survey sampling. 1965.

15. MoH(Uganda). Uganda National Policy Guidelines for HIV counselling and testing. Kampala, Uganda. 2003. 16. American Diabetes Association. Diagnosis and Classification of Diabetes Mellitus: Diabetes Care. January. 2013;36(s1).

17. David W.Hosmer, Lemeshow S. Apllied Logistic Regression. John Wiley and Sons Inc. 2000.

18. Semmens J, Rouse I, Beilin LJ, et al. Relationship of plasma HDL-cholesterol to testosterone, estradiol, and sex-hormone-binding globulin levels in men and women. Metabolism. 1983;32(5):428-432. PubMed

19. Muhs EO. Serum Cholesterol and Serum Lipids in Patients with Far Advanced Active Pulmonary Tuberculosis on Three Drugs-Preliminary Report. CHEST Journal. 1967;52(2):159-165.

20. Ghorbani HA, Rashtchizadeh N, Vatankhah A. Lipid Profiles and Lipoprotein (A) in Pulmonary Tuberculosis Patients. 2006.

21. Mupere E, Parraga IM, Tisch DJ, et al. Low nutrient intake among adult women and patients with severe tuberculosis disease in Uganda: a cross-sectional study. BMC Public Health. 2012;12(1):1 PubMed .

22. Schluger NW, WN R. The host immune response to tuberculosis. Am J Respir Crit Care Med. 1998;157(3 Pt 1):679-691.

23. Pérez-Guzmán C, Vargas M, Salas-Mártir C, et al. [Lipid profile in household contacts of patients with pulmonary tuberculosis]. Revista medica del Instituto Mexicano del Seguro Social. 2007;46(3):247-252. 